\title{
Preparation and properties of $\mathrm{Pr}^{3+} / \mathrm{Ce}^{3+}$ :YAG phosphors using triethanolamine as dispersant and $\mathrm{pH}$ regulator
}

\author{
SHIHONG TONG*, JUNYAN ZHAO and XIU WEN \\ College of Sciences of Southwest Petroleum University, Chengdu, Sichuan 610500, PR China
}

MS received 1 September 2015; accepted 28 March 2016

\begin{abstract}
Pr}^{3+} / \mathrm{Ce}^{3+}$ :YAG precursors were co-precipitated using triethanolamine as dispersant and $\mathrm{pH}$ regulator. The different dosages of triethanolamine $(D) v s$. the properties of $\mathrm{Pr}^{3+} / \mathrm{Ce}^{3+}$ :YAG phosphors were discussed. When $D=0.5 \mathrm{vol} \%$, the $\mathrm{pH}$ of titration process was controlled in the range of $\sim 7.94-8.16$ to guarantee the uniform distribution of $\mathrm{Al}, \mathrm{Y}, \mathrm{Ce}$ and $\mathrm{Pr}$ in the precursors. The relatively higher $\mathrm{pH}$ could decrease the loss of $\mathrm{Ce}$ and $\mathrm{Pr}$ in the precursors and increase the particle size of the obtained powders, which was beneficial to the enhancement of luminescent intensity. Therefore, the precursors directly converted to pure-phase YAG at $900^{\circ} \mathrm{C}$, and the phosphors calcined at $1000^{\circ} \mathrm{C}$ showed the best dispersity due to the dispersion effect of triethanolamine and the most excellent luminescent property. When $D \geq 2$ vol\%, although pure-phase YAG was detected, the emission intensity of the phosphors decreased due to the decrease of dispersity and purity. Moreover, the co-doped $\operatorname{Pr}^{3+}$ enhanced the red emission of $\mathrm{Pr}^{3+} / \mathrm{Ce}^{3+}$ :YAG phosphors.
\end{abstract}

Keywords. Co-precipitation; YAG powder; phosphor; white-light LED; ceramic.

\section{Introduction}

Yttrium aluminium garnet ( $\mathrm{YAG}, \mathrm{Y}_{3} \mathrm{Al}_{5} \mathrm{O}_{12}$ ) is an important host material due to its promising chemical stability, high-temperature mechanical properties and excellent optical properties [1-7]. Recently, Nd:YAG [1,2] as well as Yb:YAG [3] transparent ceramics for high power solid-state lasers and Ce:YAG phosphors for white LEDs [5-7] have attracted much attention. To synthesize YAG-based materials with better properties, it is required that YAG-based fine powders should have higher purity and higher dispersity [8-16].

Comparing with other ways for preparing YAG powders, such as solid-state reaction, sol-gel, hydrothermal treatment and so on, co-precipitation method is a relatively simpler and more cost-effective way [8-17]. The product synthesized by the co-precipitation method has shown good performance $[9,10]$ and great potential of applications [1,7]. The choice of precipitating agent is one of the important factors affecting the properties of YAG-based powders. The common precipitating agents for preparing YAG-based powders include ammonia water [11,12], ammonium hydrogen carbonate [8-10,13-17], and urea [18]. Compared to these precipitating agents, urea solution must be heated at high temperature for release of $\mathrm{OH}^{-}$and $\mathrm{CO}_{3}^{2-}$. High temperature and slow release of $\mathrm{OH}^{-}$and $\mathrm{CO}_{3}^{2-}$ were disadvantageous to prepare nanometre and good dispersible powders. Moreover, previous work showed that ammonium hydrogen carbonate exceeded ammonia water for the production of less-agglomerated and well-sinterable powders [10,19].

*Author for correspondence (tongshihong@ 126.com)
However, the $\mathrm{pH}$ of ammonium hydrogen carbonate solution was lower than ammonia water solution, the loss of cations in the precursors was more, which led to decrease in emission intensity [19]. In addition, for the same reason as ammonia water and urea, the different deposits for $\mathrm{Y}^{3+}, \mathrm{Al}^{3+}$ and doped rare-earth ions have different solubility products, the $\mathrm{pH}$ of co-precipitation process using ammonium hydrogen carbonate as precipitating agent also has to be controlled to guarantee that all the metal ions are simultaneously co-precipitated and evenly distributed in the precipitated slurry according to their stoichiometric proportion in YAG-based compound $[13,14]$. Otherwise, there will be impurity phases appearing during the roasting process $[8,15]$. Also, it is completely difficult to avoid agglomeration.

To solve the above problem, $\mathrm{Pr}^{3+} / \mathrm{Ce}^{3+}$ :YAG precursors were co-precipitated by using ammonium hydrogen carbonate $\left(\mathrm{NH}_{4} \mathrm{HCO}_{3}\right)$ as precipitant and triethanolamine as dispersant as well as $\mathrm{pH}$ regulator. In this study, triethanolamine was first used as dispersant and $\mathrm{pH}$ regulator for the preparation of $\mathrm{Pr}^{3+} / \mathrm{Ce}^{3+}$ :YAG phosphors. The use of triethanolamine has some advantages. First, triethanolamine could appropriately make the $\mathrm{pH}$ of precipitation process higher to guarantee the co-precipitation of Al, Y, Ce and Pr. Second, because of the buffer effect of the mixed solution of ammonium hydrogen carbonate and triethanolamine, the variation of the $\mathrm{pH}$ in the whole titration process is narrow. Thus, the $\mathrm{pH}$ is not necessarily to be controlled in the whole titration process. Third, suitable amount of triethanolamine played the role of dispersant, solving the problem of the serious segregation between particles. Moreover, the relatively higher $\mathrm{pH}$ decreased the loss of $\mathrm{Ce}$ and $\mathrm{Pr}$ in the precursors, 
which is beneficial to the enhancement of luminescent intensity. In this study, the different dosages of triethanolamine (D) $v s$. the variation of $\mathrm{pH}$ of the solution, the phase formation, dispersity, particle size and luminescent properties of $\mathrm{Pr}^{3+} / \mathrm{Ce}^{3+}$ :YAG phosphors were discussed.

\section{Experimental}

Given the chemical formula of $\left(\mathrm{Y}_{0.975} \mathrm{Ce}_{0.02} \mathrm{Pr}_{0.005}\right)_{3} \mathrm{Al}_{5} \mathrm{O}_{12}$, $\mathrm{Pr}\left(\mathrm{NO}_{3}\right)_{3} \cdot 6 \mathrm{H}_{2} \mathrm{O} \quad(99.9 \%), \quad \mathrm{Ce}\left(\mathrm{NO}_{3}\right)_{3} \cdot 6 \mathrm{H}_{2} \mathrm{O} \quad$ (99.9\%), $\mathrm{Y}\left(\mathrm{NO}_{3}\right)_{3} \cdot 6 \mathrm{H}_{2} \mathrm{O}(99.9 \%)$ and $\mathrm{NH}_{4} \mathrm{Al}\left(\mathrm{SO}_{4}\right)_{2} \cdot 12 \mathrm{H}_{2} \mathrm{O}(99.9 \%)$ were weighted in the molar ratio of 0.015:0.06:2.925:5 and dissolved in distilled water to obtain a mixed solution, in which the concentration of $\mathrm{Al}^{3+}$ was $0.1 \mathrm{M}$. The precipitating agent solution, in which the concentration of ammonium hydrogen carbonate $\left(\mathrm{NH}_{4} \mathrm{HCO}_{3}\right.$, A.R.) was $0.5 \mathrm{M}$, was evenly divided into four parts with $0,0.5,2$ and $4 \%$ by volume triethanolamine added, respectively. Then the same amount of mixed solution was dropped into the four parts of precipitating agent solution at a speed of $200 \mathrm{ml} \mathrm{h}^{-1}$, accompanied by stirring at room temperature. After titration, the suspensions were aged for $24 \mathrm{~h}$, and then filtered and washed with distilled water and alcohol. After drying the precipitates at $80^{\circ} \mathrm{C}$ for $24 \mathrm{~h}$ in a thermostatic drier, the obtained precursors were calcined at different temperatures for $2 \mathrm{~h}$.

The compositions of precipitates were investigated by the Fourier transformation infrared spectroscope (FT-IR, PerkinElmer Company, Spectrum one). The $\mathrm{pH}$ of precipitation process was monitored by a $\mathrm{pH}$ meter with an accuracy of 0.02 . The phase formation process and microstructure of $\mathrm{Pr}^{3+} / \mathrm{Ce}^{3+}$ :YAG powders were investigated by X-ray diffraction (XRD, D/max-rA model, using nickel-filtered $\mathrm{Cu}-\mathrm{K} \alpha$ radiation) and the field emission scanning electron microscope (SEM, HITACHI, S-4800). Photoluminescence (PL) spectra were measured with a Hitachi Spectra-fluorimeter (F-7000).

\section{Results and discussion}

Table 1 shows the variation of the $\mathrm{pH}$ of ammonium hydrogen carbonate solution with different dosages of triethanolamine $(D)$. The initial $\mathrm{pH}$ is the $\mathrm{pH}$ of precipitating agent solution and the final $\mathrm{pH}$ is the $\mathrm{pH}$ of the solution after titration. The $\mathrm{pH}$ of the solution was increased with increase in $D$. Because of the buffer effect of the mixed solution of ammonium hydrogen carbonate and triethanolamine, the

Table 1. Variation of the $\mathrm{pH}$ of ammonium hydrogen carbonate solution with different dosages of triethanolamine $(D)$.

Dosages of triethanolamine $(D) \quad 0$ vol\% $\quad 0.5$ vol\% 2 vol\% 4 vol\%

\begin{tabular}{lllll}
\hline Initial pH & 7.78 & 8.16 & 8.44 & 8.52 \\
Final pH & 7.46 & 7.94 & 8.14 & 8.20 \\
\hline
\end{tabular}

variation of the $\mathrm{pH}$ in the titration process was narrow, and when $D \geq 2$ vol\%, the $\mathrm{pH}$ was increased very slowly.

Figure 1 shows the XRD patterns of $\mathrm{Pr}^{3+} / \mathrm{Ce}^{3+}$ :YAG powders prepared under different experimental conditions, where the series of samples prepared under $D=0,0.5,2$ and 4 vol\% and calcined at $1000^{\circ} \mathrm{C}$ for $2 \mathrm{~h}$ are labelled by $\mathrm{Y} 1$, Y2, Y3 and Y4. It can be seen that the sample prepared under $D=0.5 \mathrm{vol} \%$ and calcined at $900^{\circ} \mathrm{C}$ for $2 \mathrm{~h}$ was completely transformed into pure YAG phase, which indicates that $\mathrm{Pr}^{3+}$ and $\mathrm{Ce}^{3+}$ successfully replaced $\mathrm{Y}^{3+}$ into the YAG lattice. As the temperature reached $1000^{\circ} \mathrm{C}$, only the crystallinity was improved. Moreover, for those samples calcined at $1000^{\circ} \mathrm{C}$ for $2 \mathrm{~h}$, except $\mathrm{Y} 1$ in which a few impure phases were detected, all the other samples were pure YAG phase. It seems that triethanolamine is beneficial to the preparation of pure-phase $\mathrm{Pr}^{3+} / \mathrm{Ce}^{3+}$ :YAG. The reason should be that the $\mathrm{pH}$ of the titration process was increased by triethanolamine to guarantee the co-precipitation of $\mathrm{Al}^{3+}, \mathrm{Y}^{3+}, \mathrm{Ce}^{3+}$ and $\mathrm{Pr}^{3+}$ and the uniform distribution of $\mathrm{Al}^{3+}, \mathrm{Y}^{3+}, \mathrm{Ce}^{3+}$ and $\mathrm{Pr}^{3+}$ in the precursors.

Figure 2 shows SEM images of Y1, Y2, Y3 and Y4. Compared with Y1 prepared under $D=0$ vol\%, Y2 prepared under $D=0.5$ vol\% shows much better dispersity, which should be attributed to the dispersion effect of triethanolamine. When $D \geq 2$ vol\%, the dispersity of these samples became worse, which should be attributed to that an excess of triethanolamine lost the role of dispersant and simultaneously led to the relatively higher $\mathrm{pH}$ resulting in the enhancement of agglomeration between particles [11]. The change of the dispersity is also proved by the particle size distribution of Y1, Y2, Y3 and Y4, as shown in figure 3. Figure 3 shows that the particle size distribution of $\mathrm{Y} 2$ is the narrowest. By comparing the average particle size of Y1, Y2, $\mathrm{Y} 3$ and Y4, as shown in table 2, it can be seen that the average particle size of $\mathrm{Y} 2$ obtained by the particle size distribution is closest to the result observed by SEM. The average particle sizes of Y1 and Y4 obtained by the particle size distribution are much different from the result observed by SEM, because

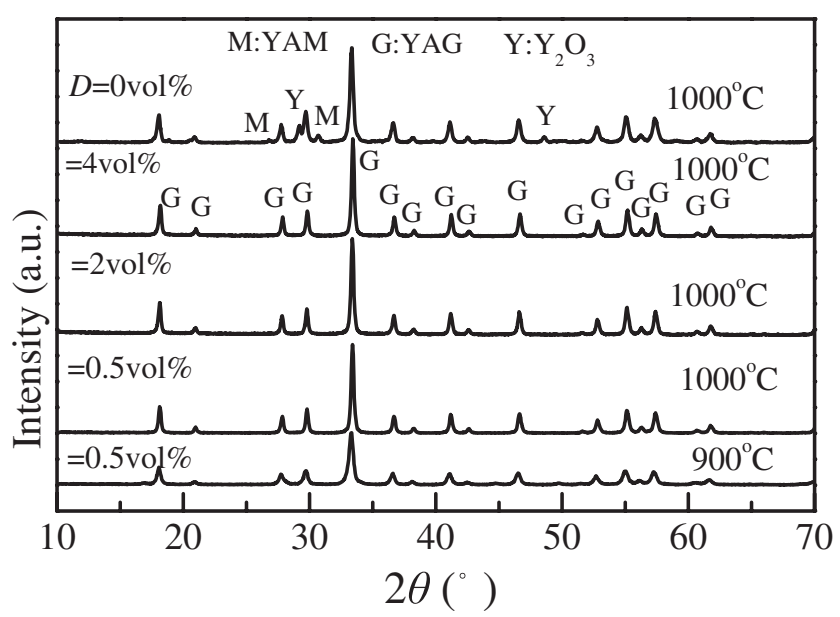

Figure 1. XRD patterns of YAG powders prepared under different experimental conditions. 

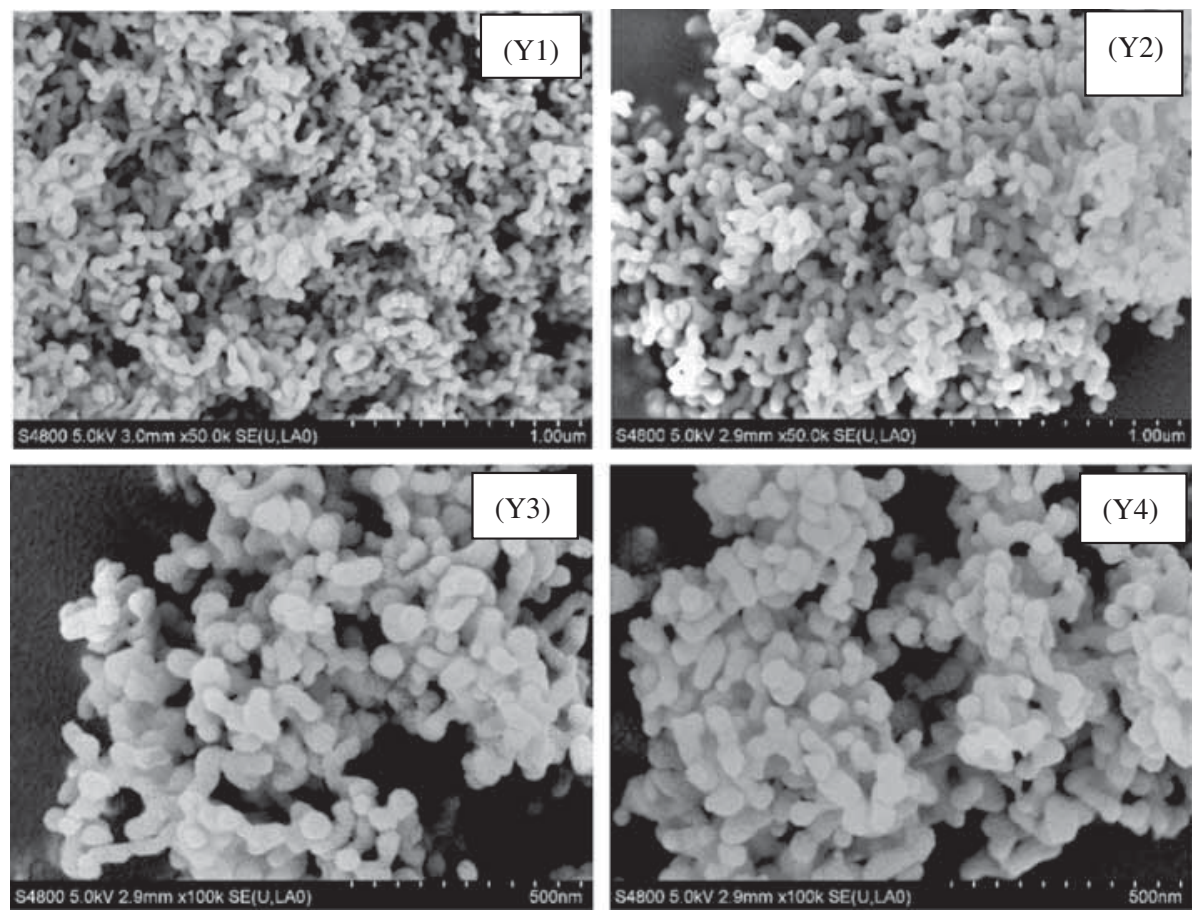

Figure 2. SEM images of Y1, Y2, Y3 and Y4.
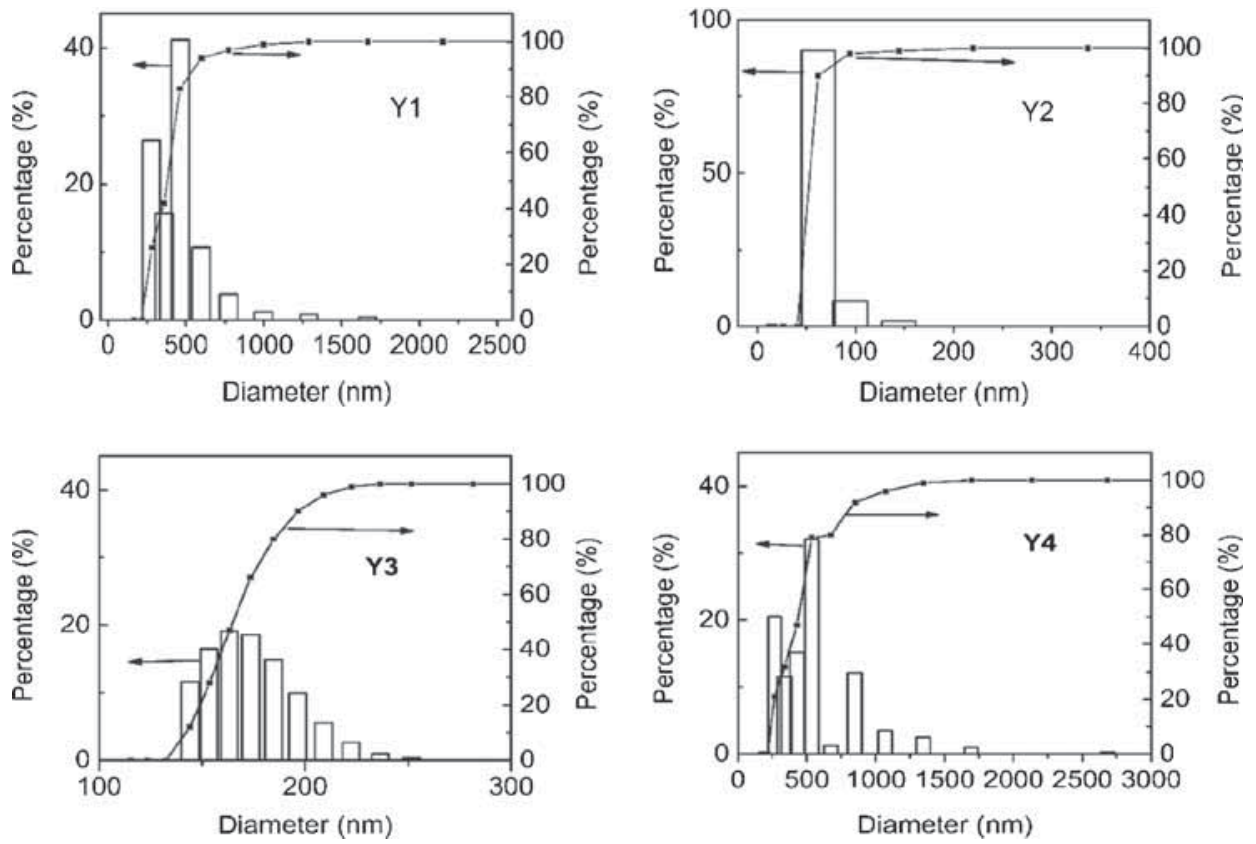

Figure 3. The particle size distribution of $\mathrm{Y} 1, \mathrm{Y} 2, \mathrm{Y} 3$ and $\mathrm{Y} 4$.

of the serious agglomeration of Y1 and Y4. It is known that the average particle size obtained by the particle size distribution should be the average size of the aggregate derived from the reunion of some particles. Therefore, from figure 3 we conclude that Y2 shows the best dispersity. In addition, as shown in figure 2, although the morphologies of all the samples are approximately spherical, the average particle size of Y2 is obviously larger than that of $\mathrm{Y} 1$, and the particle size increases slowly when $D$ is increased from 0.5 to 4 vol\%.
Furthermore, as shown in table 2, the crystallite size of Y1, $\mathrm{Y} 2, \mathrm{Y} 3$ and $\mathrm{Y} 4$ are 28.4, 36.7, 38.5 and $39.0 \mathrm{~nm}$, respectively, which was calculated by Scherrer equation from XRD data. The result also shows that the crystallite size increases gradually with increase in $D$. This can be explained by the fact that the change of particle size also is related to the change of the $\mathrm{pH}$. Because the increase of the $\mathrm{pH}$ can lead to the increase of particle size [11], the particle sizes of $\mathrm{Y} 2, \mathrm{Y} 3$ and $\mathrm{Y} 4$ are obviously larger than that of $\mathrm{Y} 1$. However, the $\mathrm{pH}$ increases 
Table 2. Average particle and crystallite sizes of Y1, Y2, Y3 and Y4 obtained by different methods.

\begin{tabular}{lcccc}
\hline Samples & Y1 (nm) & Y2 (nm) & Y3 (nm) & Y4 (nm) \\
\hline $\begin{array}{l}\text { Average particle size (SEM) } \\
\text { Average particle size }\end{array}$ & $\sim 45$ & $\sim 53$ & $\sim 55$ & $\sim 55$ \\
$\quad$ particle size distribution) & 441.5 & 67.5 & 173.0 & 542.2 \\
$\begin{array}{l}\text { Average crystallite size (XRD) } \\
\text { (28.4 }\end{array}$ & 36.7 & 38.5 & 39.0 \\
\hline
\end{tabular}

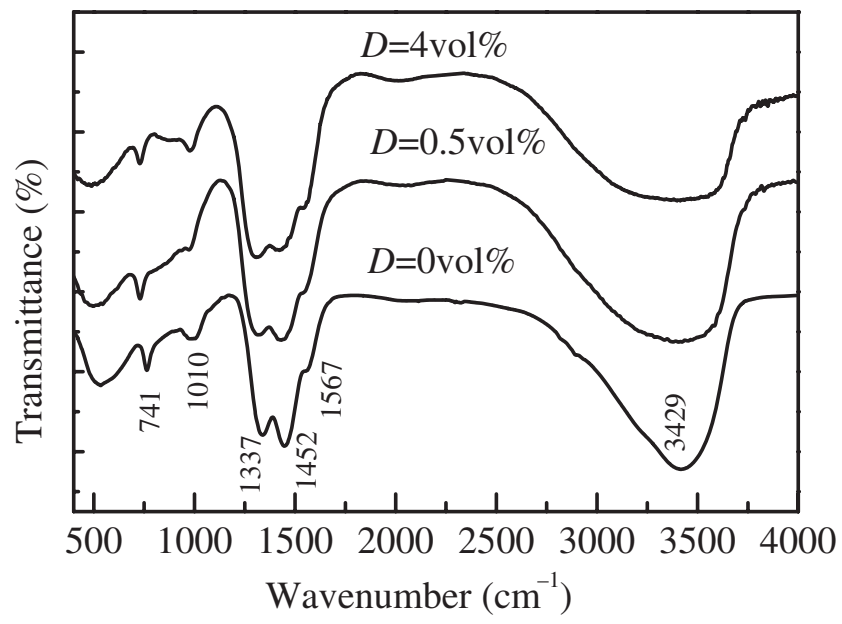

Figure 4. FT-IR spectra of the precursors synthesized by using different $D$.

slowly when $D$ is above $2 \mathrm{vol} \%$, in this case the increase of particle size is not obvious.

Furthermore, the change in the dispersity of these samples can also be explained by the FT-IR spectra of the precursors synthesized under $D=0,0.5$ and 4 vol\%, respectively, as shown in figure 4. It is seen that, the positions of main peaks whose vibrations are given by other literature [20] are the same in the three curves, which suggests that the trithanolamine could not change the main compositions of the precursors. However, it is found that the absorption peak at $\sim 3429 \mathrm{~cm}^{-1}$ gradually becomes a wider peak in the range $3000-3500 \mathrm{~cm}^{-1}$ when $D$ is increased from 0 to $4 \mathrm{vol} \%$, and the absorption intensity gradually becomes weaker, which indicates that the hydrogen bonding interaction of absorbed water is enhanced. It is supposed that the enhancement of hydrogen bonding interaction can lead to more serious agglomeration of the powders, which is consistent with the results depicted in figure 2 . However, when $D=0.5 \mathrm{vol} \%$, the powders show the best dispersity, which indicates that the agglomeration does not increase linearly with increase in $D$, simply because trithanolamine is both $\mathrm{pH}$ regulator and dispersant.

The virtue of trithanolamine is also confirmed by the PL spectra of $\mathrm{Y} 1, \mathrm{Y} 2, \mathrm{Y} 3$ and $\mathrm{Y} 4$, as shown in figure 5. It can be seen that, the emission intensity of Y1 is the weakest, while Y2 is the strongest. When $D=0.5$ vol\%, either increasing or reducing $D$ will decrease the emission intensity. According to the above analysis, the use of trithanolamine increased the $\mathrm{pH}$ of the solution, the loss of $\mathrm{Ce}$ and $\mathrm{Pr}$ in the precursors should become less with increase in $D$ [19]. The lower

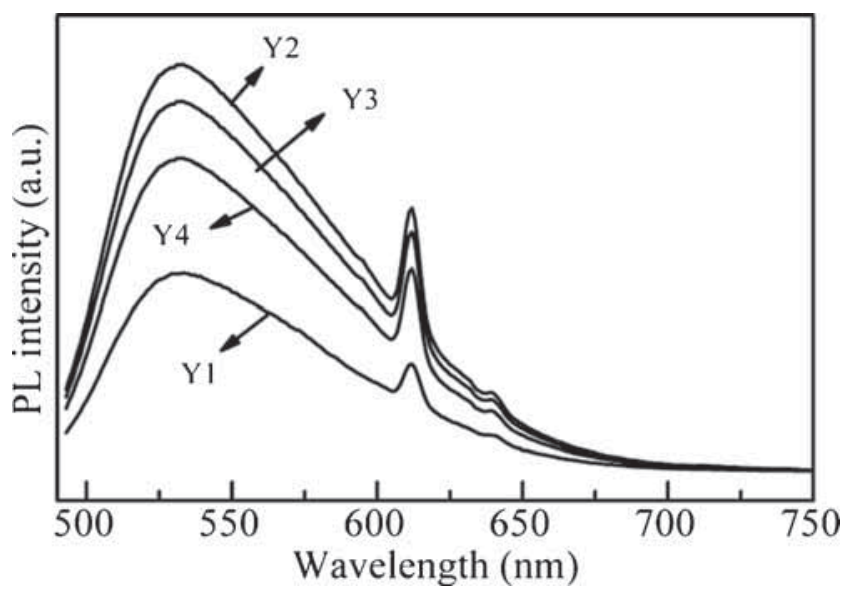

Figure 5. PL spectra of $\mathrm{Y} 1, \mathrm{Y} 2, \mathrm{Y} 3$ and $\mathrm{Y} 4\left(\lambda_{\mathrm{ex}}=470 \mathrm{~nm}\right)$.

loss of $\mathrm{Ce}$ and Pr led to increase in emission intensity. Also, the particle size of the samples prepared under $D \geq 0.5$ vol\% was bigger than under $D=0$ vol\%, the small specific area brought on less surface defects, which led to decrease the possibility of nonradiative transition [19]. In addition, the purity of the samples prepared under $D \geq 0.5 \mathrm{vol} \%$ was higher than under $D=0$ vol\%, which also led to increase in emission intensity. Therefore, the emission intensity of the samples prepared under $D \geq 0.5$ vol\% was stronger than under $D=0$ vol $\%$. While $D \geq 2$ vol\%, the emission intensity was decreased, which should be attributed to the decrease of dispersity and purity of the phosphors. When $D \geq 2$ vol\%, the higher $\mathrm{pH}$ decreased the loss of $\mathrm{Y}, \mathrm{Ce}$ and $\mathrm{Pr}$, but increased the loss of $\mathrm{Al}$ in the precursors, which led to conclude that the molar ratio of $(\mathrm{Y}+\mathrm{Ce}+\mathrm{Pr})$ : $\mathrm{Al}$ was seriously over 0.6. Although the samples prepared under $D \geq 2$ vol\% were pure-phase YAG, the purity of the powders was decreased due to the amount of secondary phases $\left(\mathrm{Y}_{2} \mathrm{O}_{3}, \mathrm{YAM}\right.$ and YAP), which were too few to be detected by the XRD equipment [13]. Thus, $D=0.5 \mathrm{vol} \%$ is beneficial to the preparation of pure-phase $\mathrm{Pr}^{3+} / \mathrm{Ce}^{3+}$ :YAG. Furthermore, as $D=0.5 \mathrm{vol} \%$, the $\mathrm{pH}$ of the titration process controlled in the range of $\sim 7.94-8.16$ is beneficial to the fabrication process of $\mathrm{Pr}^{3+} / \mathrm{Ce}^{3+}$ :YAG precursors, which is consistent with the conclusions of other literature [13].

It should also be remarked that, all curves exhibit a wellknown yellow emission broadband peaking around $530 \mathrm{~nm}$ due to the transition from the lowest $5 \mathrm{~d}$ state of $\mathrm{Ce}^{3+}$ to the $4 \mathrm{f}$ ground state of $\mathrm{Ce}^{3+}$. The intense red emission line at $612 \mathrm{~nm}$ is due to ${ }^{1} \mathrm{D}_{2} \rightarrow{ }^{3} \mathrm{H}_{4}$ of $\operatorname{Pr}^{3+}$, which indicates that the co-doped $\mathrm{Pr}^{3+}$ has enhanced the red emission of the $\mathrm{Pr}^{3+} / \mathrm{Ce}^{3+}$ :YAG phosphors. The phosphors can be used as candidate materials for the preparation of white LEDs with high colour rendering.

\section{Conclusion}

$\mathrm{Pr}^{3+} / \mathrm{Ce}^{3+}$ :YAG precursors were co-precipitated by using ammonium hydrogen carbonate $\left(\mathrm{NH}_{4} \mathrm{HCO}_{3}\right)$ as precipitant 
and triethanolamine as dispersant as well as $\mathrm{pH}$ regulator. The different dosages of triethanolamine $(D) v s$. the properties of $\mathrm{Pr}^{3+} / \mathrm{Ce}^{3+}$ :YAG phosphors were discussed. When $D=0.5 \mathrm{vol} \%$, the $\mathrm{pH}$ of the titration process was controlled in the range of $\sim 7.94-8.16$ to guarantee the uniform distribution of $\mathrm{Al}, \mathrm{Y}, \mathrm{Ce}$ and $\mathrm{Pr}$ in the precursors, and the precursors directly converted to pure YAG at about $900^{\circ} \mathrm{C}$. The precursors synthesized under $D=0$ vol\% transformed to YAG via a few impure phases at about $1000^{\circ} \mathrm{C}$. The $\mathrm{Pr}^{3+} / \mathrm{Ce}^{3+}$ :YAG phosphors synthesized under $D=0.5$ vol\% showed the best dispersity due to the role of dispersant played by triethanolamine and the best luminescent property on account of the low loss of $\mathrm{Ce}$ and $\mathrm{Pr}$, high purity, big particle size and good dispersity of the phosphors. When $D \geq 2$ vol\%, the dispersity of the powders became worse owing to the enhancement of hydrogen bonding interaction, and the emission intensity decreased due to the decrease of dispersity and purity. Moreover, the co-doped $\mathrm{Pr}^{3+}$ enhanced red emission of the $\mathrm{Pr}^{3+} / \mathrm{Ce}^{3+}$ :YAG phosphors, which can be used as candidate materials for the preparation of white LEDs with high colour rendering.

\section{Acknowledgement}

This research was supported by the Technology Fund of Southwest Petroleum University, under Grant 2013XJZ026.

\section{References}

[1] Lu J R, Song J, Prabhu M et al 2000 Japanese J. Appl. Phys. 39 L1048

[2] Yang H, Qin X P, Zhang J, Ma J, Tang D Y, Wang S W and Zhang Q T 2012 Opt. Mater. 34940
[3] Tang F, Cao Y G, Huang J Q, Liu H G, Guo W and Wang W C 2012 J. Am. Ceram. Soc. 9556

[4] Cavalli E, Esposito L, Hostasa J and Pedroni M 2013 J. Eur. Ceram. Soc. 331425

[5] Potdevin A, Chadeyron G, Boyer D and Mahiou R 2007 Physica Status Solidi C 465

[6] Nishiura S, Tanabe S, Fujioka K and Fujimoto Y 2011 Opt. Mater. 33688

[7] Wei N, Lu T C, Li F et al 2012 Appl. Phys. Lett. $101061902-$ 061902-4

[8] Zeng M, Ma Y J, Wang Y H and Pei C H 2012 Ceram. Int. 386951

[9] Tong S H, Lu T C and Guo W 2007 Mater. Lett. 61 4287

[10] Li J G, Ikegami T, Lee J H, Mori T and Yajima Y 2000 J. Eur. Ceram. Soc. 202395

[11] Wang H Z, Gao L and Niihara K 2000 Mater. Sci. Eng. A 2881

[12] Palmero P, Esnouf C, Montanaro L and Fantozzi G 2005 J. Eur. Ceram. Soc. 251565

[13] Liu W B, Zhang W X, Li J et al 2010 J. Alloy Compd. 503525

[14] Sang Y H, Lv Y, Qin H, Zhang X, Liu H and Boughton R I 2012 Ceram. Int. 381635

[15] Li J G, Ikegami T, Lee J H and Mori T $2000 \mathrm{~J}$. Am. Ceram. Soc. 83961

[16] Xu G, Zhang X D, He W et al 2006 Mater. Lett. 60962

[17] Li X, Liu H, Wang J Y, Cui H M, Zhang X D and Han F 2004 Mater. Sci. Eng. A 379347

[18] Li J S, Sun X D, Liu S H, Li X D, Li J G and Huo D 2015 Ceram. Int. 413283

[19] Zhang K, Liu H Z, Wu Y T and Hu W B 2008 J. Alloys Comp. 453265

[20] Zhao D L, Yang Q, Han Z H, Sun F Y, Tang K B and Yu F 2008 Solid State Sci. 101028 